\title{
Percepción de estudiantes de enfermería sobre el aprendizaje basado en evidencias*
}

\author{
Perception of Nursing Students about Evidence-Based Learning \\ Percepção de estudantes de enfermagem sobre a aprendizagem baseada em evidências
}

Marcia Paschoalina Volpato ${ }^{\text {a }}$
Universidade Estadual de Londrina, Brasil
volpato@uel.br

Maria Helena Larcher Caliri

Universidade de São Paulo, Brasil

Maria José Quina Galdino

Universidade Estadual do Norte do Paraná, Brasil

Júlia Trevisan Martins

Universidade Estadual de Londrina, Brasil
DOI: https://doi.org/10.11144/Javeriana.ie20-1.peea

Fecha de recepción: 26 Marzo 2016 Fecha de aprobación: 16 Noviembre 2016

\section{Resumen:}

Objetivo: revelar la percepción de estudiantes de enfermería sobre la práctica basada en evidencias como estrategia de enseñanza y aprendizaje en la prevención de úlceras por presión. Métodos: estudio cualitativo realizado con doce estudiantes de graduación en enfermería de una universidad del sur de Brasil que participaron en un workshop teórico-práctico sobre la prevención de úlceras por presión. Los datos se recabaron a través de entrevistas semiestructuradas y se sometieron a análisis temático. Resultados: se evidenció la categoría temática como la estrategia de la práctica basada en evidencias en la enseñanza-aprendizaje de úlceras por presión, con cuatro subcategorías: facilitando la correlación entre teoría y práctica y la búsqueda de soluciones; activando las competencias del enfermero; fomentando la identificación de las fases de la úlcera por presión, y revelando la importancia de la escala de Braden. Conclusión: los estudiantes destacaron que la práctica basada en evidencias fomentó un aprendizaje amplio sobre las medidas para prevenir las úlceras por presión.

Palabras clave: úlcera por presión, práctica clínica basada en evidencias, enseñanza, aprendizaje.

\section{Abstract:}

Objective: To reveal the perception of nursing students on the practice based on evidence as a teaching and learning strategy in the prevention of pressure ulcers. Methods: A qualitative study performed with 12 undergraduate nursing students of a public university in southern Brazil, who participated in a theoretical and practical workshop about the prevention of pressure ulcers. Data were collected by semi-structured interviews and submitted to thematic analysis. Results: It was noted the thematic category the strategy of evidence based on the teaching and learning of pressure ulcers practice, with four subcategories: facilitating the correlation between theory and practice and the search for solutions; awakening the nurse skills; enabling the identification of pressure ulcer stages and awakening to the importance of the Braden scale. Conclusion: The students emphasized that evidencebased practice provided a wide learning about measures to prevent pressure ulcers.

Keywords: pressure ulcer, evidence-based clinical practice, teaching, learning.

\section{Resumo:}

Objetivo: revelar a percepção de estudantes de enfermagem sobre a prática baseada em evidências como estratégia de ensino e aprendizagem na prevenção de úlceras por pressão. Métodos: estudo qualitativo realizado com 12 estudantes de graduação em enfermagem de uma universidade pública do sul do Brasil, que participaram de um workshop teórico-prático sobre a prevenção de úlceras por pressão. Os dados foram coletados por entrevistas semiestruturadas e submetidos à análise temática. Resultados: evidenciou-se a categoria a estratégia da prática baseada em evidências no ensino-aprendizado de úlceras por pressão, com quatro subcategorias: facilitando a correlação entre teoria e prática e a busca por soluções; despertando para as competências do enfermeiro; propiciando a identificação dos estágios da úlcera por pressão e despertando para importância da escala de Braden. Conclusão: os

Notas de autor 
estudantes destacaram que a prática baseada em evidências propiciou uma aprendizagem ampla sobre as medidas para se prevenir as úlceras por pressão.

Palavras-chave: úlcera por pressão, prática clínica baseada em evidências, ensino, aprendizagem.

\section{Introducción}

La formación del enfermero como profesional de un equipo multidisciplinario de salud debe ser dinámica y provista de reflexiones, a efectos de formar personas con competencia y perfil, para provocar los cambios necesarios en las prácticas sanitarias. En este contexto, las enseñanzas en las carreras del área sanitaria deben acercarse a la realidad social en la formación de un profesional activo, es decir, aquel que busque sus conocimientos por medio de un aprender a conocer, aprender a hacer, aprender a ser y a convivir y, por tanto, adquirir competencias para atender la salud de las personas, en el marco de la integridad, de la calidad, de la eficiencia y de la capacidad de resolución $[1,2,3]$.

De este modo, es imprescindible que las carreras de graduación fomenten en los estudiantes una formación enfocada en las competencias del cuidado integral; en otras palabras, una asistencia holística en forma de conocimiento, habilidades y actitudes. Para ello, se debe estimular el desarrollo del pensamiento crítico que servirá como base para la toma de decisión clínica y para la práctica basada en evidencias (PBE), lo que significa emplear los resultados de las investigaciones científicas para orientar las decisiones prácticas y para fomentar una mejor calidad en el cuidado, el tratamiento efectivo, la reducción de costos y la satisfacción profesional $[4,5]$.

La PBE implica "el uso consciente, explícito y cabal de la mejor evidencia actual para toma de decisión sobre el cuidar individual" del paciente [6]. De esta forma, con la PBE se establecen prioridades, se invierte cada vez más en el diagnóstico clínico de las personas y se libera tiempo para iniciativas relativas a la búsqueda de resultados de investigaciones, así como desarrollarlas [7].

La incorporación de la PBE a la formación académica de los enfermeros puede desarrollarse en cinco etapas: en la primera se deben incentivar el cuestionamiento crítico y la curiosidad intelectual sobre la práctica profesional, estimulando al alumno a pensar más allá de lo obvio y a identificar explicaciones alternativas. En la segunda, el estudiante deberá aprender a formular una buena pregunta clínica, que lo ayudará a aclarar los elementos esenciales de la cuestión. En la tercera etapa se enseña a los estudiantes a buscar en las bases de datos nacionales e internacionales las bibliografías de las mejores evidencias científicas relativas a la pregunta planteada. En la cuarta se deben ofrecer elementos al alumno para que pueda evaluar críticamente las evidencias encontradas, teniendo en cuenta la validez y confiabilidad metodológicas, además de su aplicabilidad clínica, así como la aplicación en la práctica de los hallazgos elegidos. La quinta y última etapa consiste en evaluar los resultados; aquí el profesor destaca la importancia del seguimiento y evaluación de los resultados de la intervención, con el fin de identificar si la intervención surtió el efecto esperado y si los resultados fueron consistentes o discrepantes con la literatura [8].

El empleo de la PBE en la graduación en enfermería es una estrategia de enseñanza-aprendizaje que pueden emplear profesores y tutores que estén enseñando cualquier contenido de la práctica profesional de enfermería [8], sobre todo los problemas usuales presentes cotidianamente en esta práctica. En este sentido, un objeto que despierta bastante por parte de la enfermería es el escenario de la prevención de las úlceras por presión, que es una importante causa de morbimortalidad de los individuos bajo sus cuidados, principalmente en el ambiente hospitalario $[9,10]$.

Además, el cuidado y prevención de heridas forma parte de lo cotidiano del enfermero, debe ejecutarse en el contexto del proceso de enfermería y seguir los principios de seguridad del paciente, con una asistencia segura de salud a la persona, incluyendo la prevención de úlceras por presión [11,12]. Para que esto se haga realidad, la implementación de intervenciones basadas en evidencias científicas es esencial, de manera que se controlen los factores individuales de riesgo y, de esta manera, evitar el surgimiento de la úlcera o permitir su resolución. 
Sin embargo, varios estudios han venido demostrando [13,14] que el empleo de la PBE en el abordaje de úlceras por presión en pacientes de riesgo todavía es poco conocido por los graduandos y profesionales de enfermería; hay déficits de conocimiento sobre los aspectos de cuidado y prevención, además de una práctica rodeada de mitos, tradiciones y "sentido común".

Ante estas consideraciones, surge la siguiente pregunta: ¿cuál es la percepción de los estudiantes de enfermería sobre la PBE como estrategia de enseñanza y aprendizaje en la prevención de úlceras por presión? Para responder a esta indagación, se persiguió con este estudio revelar la percepción de estudiantes de enfermería sobre la PBE como estrategia de enseñanza y aprendizaje en la prevención de úlceras por presión. En el presente estudio cabe hacer hincapié en qué se entiende sobre percepción, aquella que implica la subjetividad, sentimientos, juicios de valor de las personas, es decir, los individuos en su singularidad, teniendo en cuenta sus creencias, culturas y modus vivendi [15].

\section{Métodos}

Esta investigación fue un estudio descriptivo-exploratorio, con abordaje cualitativo realizado en un curso de graduación en enfermería de una universidad pública de la región sur de Brasil, que emplea el currículo integrado desde el 2000. El universo objeto de estudio fue el de estudiantes del segundo año del referido curso, matriculados en el módulo Prácticas del Cuidar, en el que se aborda la enseñanza de las úlceras por presión. Los criterios de inclusión de los participantes fueron: ser estudiante regular del curso y aceptar libremente participar del estudio, tras haber sido explicitados los objetivos y métodos de investigación.

Con base en estos criterios de elegibilidad, 12 estudiantes participaron de esta investigación. En el periodo que va de octubre a noviembre del 2012, estos estudiantes frecuentaron un workshop con duración de 60 horas, distribuidas en 4 encuentros ( 16 horas) teóricos y 14 encuentros ( 44 horas) para actividades prácticas en el ambiente hospitalario. El workshop se diseñó pensando en temas relacionados con la PBE aplicada a la prevención de úlceras por presión y directrices de la National Pressure Ulcer Advisory Panel, European Pressure Ulcer Advisory Panel y la Pan Pacific Pressure Injury Alliance (NPUAP/EPUAP/PPPIA) [14], según se explica en la tabla 1. 
TABLA 1

Encuentros, temática y técnicas del workshop de prevención de úlceras por presión basada en evidencias científicas

\begin{tabular}{|c|c|c|}
\hline Encuentro & Temática & Técnica \\
\hline 1 & $\begin{array}{l}\text { PBE: conceptos, aplicabilidad y } \\
\text { relevancia. Cinco etapas de la } \\
\text { PBE. Clasificación de las } \\
\text { evidencias científicas }\end{array}$ & $\begin{array}{l}\text { - Clase expositiva y dialogada } \\
\text { - Simulación de búsquedas en las bases } \\
\text { de datos } \\
\text { - Lectura dirigida de artículos científicos }\end{array}$ \\
\hline 2 & $\begin{array}{l}\text { Anatomía y fisiología de la piel. } \\
\text { Evaluación de la piel/ sistema } \\
\text { tegumentario. Fisiopatología de la } \\
\text { úlcera por presión. Regiones }\end{array}$ & $\begin{array}{l}\text { - Clase expositiva y dialogada } \\
\text { - Estudios de caso y discusión de los } \\
\text { casos en grupo }\end{array}$ \\
\hline 3 & $\begin{array}{l}\text { Factores de riesgo para el } \\
\text { desarrollo de la úlcera por } \\
\text { presión. Evaluación del riesgo por } \\
\text { la escala de Braden. Directrices }\end{array}$ & $\begin{array}{l}\text { - Simulación en laboratorio } \\
\text { - Discusión de artículos científicos con } \\
\text { evidencias de prevención para úlcera por } \\
\text { presión }\end{array}$ \\
\hline 4 & $\begin{array}{l}\text { Diagnóstico de enfermería para la } \\
\text { prevención de la úlcera por } \\
\text { presión. Intervenciones de } \\
\text { enfermería para prevención de la } \\
\text { úlcera por presión de acuerdo } \\
\text { con la NPUAP/EPUAP/PPPIA }\end{array}$ & $\begin{array}{l}\text { - Clase expositiva y dialogada } \\
\text { - Discusión de métodos para reducir la } \\
\text { incidencia de úlceras por presión: } \\
\text { protocolos } \\
\text { - Discusión de artículos científicos con } \\
\text { evidencias de prevención para úlcera por } \\
\text { presión }\end{array}$ \\
\hline 5 a 14 & $\begin{array}{l}\text { Prácticas de evaluación de riesgo } \\
\text { en los pacientes internados en el } \\
\text { hospital universitario; } \\
\text { levantamiento de casos de úlcera } \\
\text { por presión; búsqueda y } \\
\text { aplicación de las evidencias para } \\
\text { cada caso }\end{array}$ & $\begin{array}{l}\text { - Observación de la práctica con } \\
\text { posterior discusión } \\
\text { - Desarrollo e implementación por el } \\
\text { estudiante de un plan de cuidados para el } \\
\text { paciente en riesgo de desarrollo de úlcera } \\
\text { por presión basado en evidencias } \\
\text { científicas }\end{array}$ \\
\hline 15 & $\begin{array}{l}\text { Evaluación del workshop y } \\
\text { clausura }\end{array}$ & $\begin{array}{l}\text { - Mesa redonda con la presencia del } \\
\text { equipo multiprofesional del hospital para } \\
\text { discusión de los aspectos preventivos de la } \\
\text { úlcera por presión } \\
\text { - Evaluación oral con los alumnos } \\
\text { participantes }\end{array}$ \\
\hline
\end{tabular}

Fuente: elaboración propia

Finalizado el workshop, se realizaron entrevistas individuales con estos estudiantes, en diciembre de 2012. Para la recolecta de datos se empleó el instrumento semiestructurado, a efectos de caracterizar a los participantes, y para revelar el objeto de estudio se utilizó la siguiente pregunta orientadora: ¿cuál es su percepción sobre la PBE como estrategia de enseñanza y aprendizaje empleada en el módulo Prácticas del 
Cuidar para la prevención de úlceras por presión? Las entrevistas se llevaron a cabo en salas reservadas de la universidad, tuvieron una duración de treinta minutos, se grabaron en audio digital y, posteriormente, se transcribieron íntegramente, después de finalizadas. Se destaca que la convergencia de datos [16], es decir, la repetición de las hablas se produjo en la décima entrevista, aunque se optó por entrevistar a la totalidad de los participantes del workshop, o sea, doce estudiantes.

Para el análisis de las hablas provenientes de las entrevistas se optó por la técnica del análisis de contenido, específicamente al análisis temático, que se configura en un conjunto de técnicas de análisis de las comunicaciones con el objetivo de obtener, de manera sistemática y objetiva, la descripción del contenido de los mensajes e indicadores que permiten la inferencia de conocimientos en lo que respecta a las condiciones de producción o recepción de estos mensajes [16].

De esta forma, con el fin de buscar elementos para comprender la percepción de estudiantes de enfermería sobre la PBE como estrategia de enseñanza y aprendizaje en la prevención de úlceras por presión se adoptaron los siguientes procedimientos:

- El primero correspondió al preanálisis, en el cual se transcribieron los registros. La lectura se realizó de modo fluctuante con organización del corpus, considerando los principios de exhaustividad, representatividad, homogeneidad y adecuación, a fin de volver a los presupuestos y objetivos.

- El segundo fue la descripción analítica, o sea, la explotación del material propiamente dicho, con la finalidad de alcanzar el núcleo de comprensión y clasificar las categorías que representaron las características que surgieron. Se realizaron los recortes y la elección de las unidades de registro, seleccionando y codificándolos, ante la convergencia con el fenómeno. Así, se clasificaron las unidades temáticas, es decir, hubo una categorización semántica.

- Finalmente, se trataron e interpretaron los resultados, o sea, se realizaron las inferencias e interrelaciones con el cuadro teórico alrededor de nuevas dimensiones interpretativas [16].

El Comité de Ética en Investigación de la universidad, bajo Parecer 296/2011 aprobó el proyecto. Se garantizó el anonimato de los participantes, identificando las hablas por la letra $\mathrm{E}$, seguida del número secuencial de la entrevista.

\section{Resultados}

De los 12 estudiantes participantes de este estudio, 9 pertenecían al sexo femenino, y 3 , al masculino, con edades entre los 19 y los 31 años.

El análisis de las hablas de las entrevistas llevó a construir una categoría temática denominada: estrategia de la PBE en la enseñanza-aprendizaje de úlceras por presión, con cuatro subcategorías: 1) facilitando la correlación entre teoría y práctica y la búsqueda por soluciones; 2) despertando para las competencias del enfermero; 3) fomentando la identificación de las fases de la úlcera por presión, y 4) despertando para la importancia de la escala de Braden.

\section{Subcategoría 1. Facilitando la correlación entre teoría y práctica y la búsqueda de soluciones}

Los estudiantes verbalizaron la utilización de la estrategia de la PBE, lo que permitió una efectiva correlación entre teoría y práctica sobre la temática úlcera por presión. Según se puede observar en las hablas:

Yo pude relacionar la teoría con la práctica. Me gustó mucho porque conseguimos aprender más y consolidarlo más. Sabe, ver las úlceras en vivo y en directo facilitó que consiguiera una correlación de la teoría con la práctica y quedó más interesante cuando fuimos a seguir los pasos de la PBE para buscar soluciones para los pacientes. (E2) 
Bajo mi punto de vista, fue un aprendizaje en el que la relación teoría-práctica de hecho se produjo. Cuando vemos en la práctica y buscamos solucionar los problemas del paciente basados en evidencias científicas y no solo lo visto en clase, es estupendo puesto que se puede relacionar la teoría con la práctica. (E7)

\section{Subcategoría 2. Despertando para las competencias del enfermero}

Los estudiantes se dieron cuenta de que es preciso competencia, conocimiento y habilidad para evaluar los riesgos para el desarrollo de úlceras por presión en los pacientes, y ello es una de las competencias del enfermero. Destacaron, además, la importancia de saber realizar la prescripción de enfermería con las intervenciones apropiadas para la prevención de úlcera por presión, y que la PBE fue esencial para este despertar, lo que se puede verificar en lo siguiente discurso:

La PBE fue fundamental para que pudiera conocer cuáles son las competencias del enfermero, o mejor, de la enfermería en el contexto de la úlcera por presión y más allá del contexto. Es muy importante saber nuestras competencias y ejercerlas, si no vamos a perder espacio frente a otros profesionales. Es esencial que el enfermero sepa identificar los pacientes que tienen riesgo de úlcera por presión [...] A veces [nos] enfocamos en el diagnóstico médico: en el traumatismo cráneo-encefálico, en la fractura, en el cáncer, y se olvida la prevención de la úlcera por presión, que forma parte de nuestra competencia en el cuidado integral. Creo que es esencial que el enfermero sepa cómo identificar a los pacientes que tienen riesgo de úlcera por presión, hacer diagnóstico y prescripción de enfermería basada en evidencias científicas. (E1)

\section{Subcategoría 3. Facilitando la identificación de las fases y riesgos de la úlcera por presión}

Por medio de las hablas se reveló que los estudiantes percibieron que la adopción de la estrategia de la PBE fue importante para el conocimiento y desarrollo de la habilidad para identificar las fases de la úlcera por presión. Según se identificó en las expresiones:

Mi aprendizaje fue estupendo. Ahora sé identificar las cuatro fases de úlcera por presión. La PBE fue esencial para ello, puesto que lo veíamos en la práctica e íbamos a estudiar en teoría, o viceversa. Adquirí mucha más habilidad para comprender la úlcera por presión. (E10)

\section{Subcategoría 4. Despertando a la importancia de la Escala de Braden}

La Escala de Braden, como apoyo a la evaluación del riesgo de úlcera por presión, puede notarse en las hablas de los estudiantes cuando afirman que la escala es una herramienta esencial para evaluar el riesgo de úlcera por presión de los pacientes y debe emplearse por los enfermeros en su actividad laboral cotidiana:

La Escala de Braden es la base para saber hacia dónde debemos concentrarnos. Evalúa la humedad, es fácil de aplicar, ahorra tiempo, nos da seguridad y también para el paciente. Los enfermeros deberían emplearlo en su día a día. (E4)

\section{Discusión}

La asistencia de enfermería debe prestarse en consonancia con las directrices nacionales e internacionales de seguridad al paciente, en que los riesgos de daños innecesarios asociados al cuidado de salud deben reducirse a lo mínimo aceptable. De esta manera, la adopción de medidas de prevención para la úlcera por presión reducirá las tasas de incidencia y la prevalencia y se constituirá en indicador de la calidad de asistencia de enfermería [17].

En este estudio, los estudiantes evidenciaron que la estrategia de enseñanza basada en la PBE fomentó un aprendizaje sólido sobre la úlcera por presión, ya que fue posible establecer una adecuada relación entre teoría y práctica. Se considera que es un hallazgo relevante, puesto que la laguna entre teoría y práctica está 
ampliamente documentada y relatada en la literatura sobre la enseñanza y la práctica profesional de enfermería y visualizada como un complejo problema en este ámbito [18,19]. En este sentido, algunos autores [20] afirman que teoría y práctica deben trabajarse de forma concomitante e interdependiente, es decir, no pueden ser desvinculadas, puesto que la práctica no es solamente la aplicación de la teoría, sino una verdad absoluta. La teoría se elabora para proporcionar las respuestas a las reales necesidades identificadas a partir de la práctica.

Se destaca que la PBE va más allá de la relación teoría y práctica, ya que permite a los profesionales la búsqueda por el perfeccionamiento por medio del agrupamiento de los datos de muchas investigaciones desarrolladas con una buena calidad metodológica sobre un determinado asunto, así como volverse una herramienta eficaz en la labor del enfermero [21].

También se identificó que esta estrategia de enseñanza consiguió despertar las competencias del enfermero frente a la prevención de las úlceras por presión. Los estudiantes destacaron la importancia del conocimiento y habilidad para diagnosticar e intervenir a los pacientes en riesgo. Estos atributos destacados como relevantes son elementos constitutivos de la competencia profesional, es decir, conocimientos, habilidades, experiencias, actitudes, autonomía en la toma de decisiones y valores personales empleados adecuadamente en favor de las diversas demandas del contexto del trabajo en sanidad [22].

En otras palabras, tales competencias desembocan en la tríada: saber, saber hacer y saber ser. Este conjunto se constituye en saberes teóricos necesarios para entender el objeto que se va a cuidar, basado en evidencias y con el fin de consolidar las mejores prácticas en la asistencia al ser humano. El saber hacer se refiere a la implementación por medio de procedimientos y protocolos validados y dirigidos hacia las "buenas prácticas" [23].

En este caso, los estudiantes también destacaron que forma parte de la competencia del enfermero la prescripción individualizada de medidas de prevención basadas en evidencias científicas, asociado a una mirada holística sobre el paciente, que va más allá del énfasis al diagnóstico médico y de la enfermedad.

En un estudio que validó intervenciones de la clasificación de enfermería de la Nursing Interventions Classification para pacientes con diagnóstico de riesgo de úlcera por presión, se evaluó que, a pesar de que la referida guía presentó cerca de 51 intervenciones para el diagnóstico de integridad de la piel afectada, apenas 9 se aplicaban al contexto de las úlceras por presión; por ello es importante la base de estos cuidados para estos individuos en estudios clínicos, que hayan comprobado la eficacia de tales intervenciones [24]. Además, la evaluación de riesgo individualizada y una atención a los pacientes de forma integral son condiciones que maximizan la prevención [25].

Los académicos participantes del presente estudio consiguieron caracterizar correctamente las fases de desarrollo de la úlcera por presión y citaron algunas situaciones de riesgo. Una investigación con 68 graduandos de enfermería de una institución de enseñanza superior pública sobre el cuidado a los pacientes con heridas demostró que apenas el 17,6\% de los estudiantes relató ser capaz de identificar las fases de las úlceras por presión; el $51,5 \%$ respondió que no evalúan la piel de los pacientes en el campo de la práctica, y el $64,7 \%$ no sabía qué evalúa la Escala de Braden. Estos resultados indicaron que el conocimiento de los alumnos era bajo y sugirieron que necesitan adoptarse nuevas metodologías en la enseñanza de abordaje de la úlcera por presión [26].

Otro estudio realizado con 60 estudiantes de enfermería para determinar el conocimiento y actitudes en relación con la prevención de la úlcera por presión reveló que estos, a pesar de tener una actitud positiva, presentaron un déficit de conocimiento en los aspectos referentes a la prevención y que hay un vacío en el conocimiento de la prevención de la úlcera por presión [27].

La Escala de Braden es uno de los instrumentos más conocidos y utilizados en el mundo para evaluar el riesgo de desarrollo de las úlceras por presión, indispensable para su involución y prevención. Es una herramienta que proporciona evidencias para fundamentar los cuidados de enfermería, justifica la selección de problemas y dirige las actividades que desarrolle el equipo de enfermería. Además, permite registrar los 
cuidados con las lesiones, al contribuye a la continuidad y visibilidad de actuación de la enfermería de forma práctica y científica $[28,29,30]$.

\section{Conclusión}

Los estudiantes destacaron que la PBE fomentó un aprendizaje amplio sobre las medidas para prevenir las úlceras por presión. Esta práctica permitió correlacionar la teoría con la práctica y, así, buscar soluciones para los pacientes portadores de úlcera por presión. Hubo un despertar de las competencias del enfermero frente a la prevención de este problema, así como favoreció la identificación de las fases y riesgos para úlcera por presión y tratamientos adecuados, y despertó para la importancia de la utilización de la escala de Braden.

\section{Referencias}

1. Corbellini VL, Santos BRL, Ojeda BS, Gerhart LM, Eidt OR, Stein SC, et al. Linkages and challenges in the training of professional nurses. Rev Bras Enferm [internet]. 2010 [citado 12 ene 2016];63(4):555-60. Disponible en: h ttp://www.scielo.br/pdf/reben/v63n4/09.pdf

2. Miltre SM, Siqueira-Batista R, Girardi-de-Mendonça JMG, Morais-Pinto NM, Porto-Pinto C, Moreira T, et al. Active teaching-learning methodologies in health education: current debates. Ciênc Saúde Coletiva [internet]. 2008 [citado 18 ene 2016];13(Suppl 2):2133-44. Disponible en: http://www.scielo.br/pdf/csc/v13s2/v13s2a 18.pdf

3. Gómez AG. La formación integral de estudiantes de enfermería. Investig Enferm Imagen Desarr [internet]. 2013 [citado 18 mar 2016];15(2):153-72. Disponible en: http://revistas.javeriana.edu.co/index.php/ imagenydesarrollo/article/view/7075

4. Rabeh SAN, Gonçalves, MBB, Caliri MHL, Nogueira PC, Miazaki MY. Construction and validation of a virtual educational module for topical treatment of chronic wounds. Rev Enferm UERJ. 2012;20(Esp 1):603-8.

5. Melnyk BM, Fineout-Overholt E, Stillwell SB, Williamson KM. Evidence-based practice, step by step: The seven steps of evidence-based practice. Am J Nurs [internet]. 2010 [citado 17 ene 2016];110(1):51-3. Disponible en: http://journals.lww.com/ajnonline/Fulltext/2010/01000/Evidence_Based_Practice_Step_by_Step_The_ Seven.30.aspx

6. Atallah AN, Castro AA. Evidências para melhores decisões clínicas. São Paulo: Centro Cochrane do Brasil; 1998.

7. Domenico EBL, Ide CAC. Evidence based nursing: principles and applicability. Rev Latino-Am Enfermagem [internet]. 2003 Feb [citado 2016 sep 11];11(1):115-8. Disponible en: http://www.scielo.br/pdf/rlae/v11n1/ 16568.pdf

8. Winters C, Echeverri R. Teaching strategies to support evidence based education. Crit Care Nurse [internet]. 2012 [citado 17 ene 2016];32:47-54. Disponible en: http://ccn.aacnjournals.org/content/32/3/49.full.pdf + html

9. Rolim JA, Vasconcelos JMB, Caliri MHL, Santos IBC. Prevention and treatment of pressure ulcers in the daily lives of intensivist nurse. Rev Rene [internet]. 2013 [citado 18 ene 2016];14(1):148-57. Disponible en: http://www .revistarene.ufc.br/revista/index.php/revista/article/view/336/pdf

10. World Health Organization. World Alliance for Patient Safety. Summary of the evidence on patient safety: implications for research. The Research Priority Setting Working Group of the World Alliance for Patient Safety. Geneva: World Health Organization; 2008. [citado 2015 Jun 5]. Disponible en: http://www.who.int/patients afety/information_centre/Summary_evidence_on_patient_safety.pdf

11. Resolução COFEN 501/2015, que regulamenta a competência da equipe de enfermagem no cuidado as feridas e dá outras providências [internet]. [citado 2015 jun 5]. Disponible en: http://www.cofen.gov.br/wp-content/u ploads/2015/12/ANEXO-Resolu\%C3\%A7\%C3\%A3o501-2015.pdf 
12. Miyazaki MY, Caliri MHL, Santos CB. Knowledge on pressure ulcer prevention among nursing professionals. Rev Latino-Am Enferm [internet]. 2010 [citado 12 ene 2016];18(6):1203-11. Disponible en: http://www.scielo.b r/pdf/rlae/v18n6/22.pdf

13. Santos AAR, Medeiros ABA, Soares MJGO, Costa MML. Nursing students' knowledge of wound evaluation and treatment. Rev Enferm UERJ. 2010;18(4):547-52.

14. National Pressure Ulcer Advisory Panel, European Pressure Ulcer Advisory Panel and Pan Pacific Pressure Injury Alliance. Prevention and treatment of pressure ulcers: Quick reference guide. Cambridge Media: Osborne Park, Western Australia; 2014. [citado 2015 jun 5]. Disponible en: http://www.npuap.org/wp-content/uploads/201 4/08/Updated-10-16-14-Quick-Reference-Guide-DIGITAL-NPUAP-EPUAP-PPPIA-16Oct2014.pdf

15. Almeida MAB, Gutierrez GL, Marques R. Qualidade de vida: definição, conceitos e interfaces com outras áreas de pesquisas. São Paulo: Escola de Artes, Ciências e Humanidades; 2012.

16. Bardin L. Análise de conteúdo. Lisboa: Edições 70; 2011.

17. Agência Nacional de Vigilância Sanitária do Brasil. Assistência segura: uma reflexão teórica aplicada à prática [internet]. Brasília; 2013. [citado 2015 jun 5]. Disponible en: http://www20.anvisa.gov.br/segurancadopacien te/images/documentos/livros/Livro1-Assistencia_Segura.pdf

18. Hatlevik IKR. The theory-practice relationship: reflective skills and theoretical knowledge as key factors in bridging, the gap between theory and practice in initial nursing education. J Ad Nurs [internet]. 2012 [citado 12 ene 2016];68(4):868-77. Disponible en: http://onlinelibrary.wiley.com/doi/10.1111/j.1365-2648.2011.05 789.x/epdf

19. Barlem JGT, Lunardi VL, Barlem ELD, Bordignon SS, Zacarias CC, Filho WDL. Weaknesses, strengths and challenges in the nurse's education. Esc Anna Nery [internet]. 2012 [citado 18 ene 2016];16(2):347-53. Disponible en: http://www.scielo.br/pdf/ean/v16n2/20.pdf

20. Garanhani ML, Alves E, Almeida EFP, Araújo LDS. Princípios norteadores do projeto pedagógico do currículo integrado do curso de Enfermagem. En: Kikuchi ME, Guariente MHDM, orgs. Currículo integrado: a experiência do curso de Enfermagem da Universidade Estadual de Londrina. Londrina: UEL; 2014. p. 81-96.

21. Pedrolo E, Danski MTR, Mingorance P, Lazzari LSM, Méier MJ, Crozeta K. A prática baseada em evidências como ferramenta para prática profissional do enfermeiro. Cogitare Enferm [internet]. 2009 [citado 11 sep 2016];14(4):760-3. Disponible en: http://revistas.ufpr.br/cogitare/article/view/16396/10875

22. Camelo SHH, Angerami ELS. Professional competence: The construction of concepts, strategies developed by health services and implications for nursing. Texto Contexto Enferm [internet]. 2013 [citado 18 ene 2016];22(2):552-60. Disponible en: http://www.scielo.br/pdf/tce/v22n2/v22n2a34.pdf

23. Balsanelli AP, Feldman LB, Ruthes RM, Cunha ICKO. Competências gerenciais: desafio para o enfermeiro. São Paulo: Martinari; 2008.

24. Bavaresco T, Lucena AF. Nursing Intervention Classifications (NIC) validated for patients at risk of pressure ulcers. Rev Latino-Am Enfermagem [internet]. 2012 [citado 12 ene 2016];20(6):1109-16. Disponible en: http://ww w.scielo.br/pdf/rlae/v20n6/13.pdf

25. Dantas ALM, Araújo JDB, Ferreira PC, Valença CN, Diniz KD, Lira ALBC. Pressure ulcers prevention according to the intensivist nursing professional perspective. Rev Enferm UFPE. 2013;7(1):706-12.

26. Ferreira AM, Rigotti MA, Pena SB, Paula DS, Ramos IB, Sasaki VDM. Knowledge and practice of nursing students about caring for patients with wounds. Esc Anna Nery [internet]. 2013 [citado 12 ene 2016];17(2):211-9. Disponible en: http://www.scielo.br/pdf/ean/v17n2/v17n2a02.pdf

27. Cullen Gill E, Moore Z. An exploration of fourth-year undergraduate nurses' knowledge of and attitude towards pressure ulcer prevention. J Wound Care [internet]. 2013 [citado 12 ene 2016];22(1):618-22. Disponible en: h ttp://www.magonlinelibrary.com/doi/full/10.12968/jowc.2013.22.11.618

28. Serpa LF, Santos VLCG, Campanili TCGF, Queiroz M. Predictive validity of the Braden Scale for pressure ulcer risk in critical care patients. Rev Latino-Am Enfermagem [internet]. 2011 [citado 12 ene 2016];19(1):50-7. Disponible en: http://www.scielo.br/pdf/rlae/v19n1/08.pdf 
29. Raju D, Su X, Patrician PA, Loan LA, McCarthy MS. Exploring factors associated with pressure ulcers: A data mining approach. Int J Nurs Stud [internet]. 2015 [citado 18 ene 2016];52(1):102-11. Disponible en: http://w ww.journalofnursingstudies.com/article/S0020-7489(14)00205-3/fulltext

30. Brunet RNM, Kurcgant P. The incidence of pressure ulcers after the implementation of a prevention protocol. Rev Latino-Am Enfermagem [internet]. 2012 [citado 17 ene 2016];20(2). Disponible en: http://www.scielo.br/pd f/rlae/v20n2/pt_16

\section{Notas}

* Artículo de investigación.

\section{BY}

\section{Información adicional}

Cómo citar: Volpato M, Caliri MH, Galdino MJ, Martins J. Percepción de estudiantes de enfermería sobre el aprendizaje basado en evidencias. Investig Enferm Imagen Desarr. 2018;20(1):xx-xx. https://doi.org/10. 11144/Javeriana.ie20-1.peea 